\title{
Causal Model of Work Engagement among Registered Nurses and Licensed Practical Nurses Working in Long-Term Care Contexts in Japan
}

\author{
Chiaki Kinouchi \\ Department of Nursing \\ Iwate University of Health and Medical Sciences \\ Iwate, Japan \\ kinouchi@iwate-uhms.ac.jp \\ Yuko Takayama \\ Faculty of Healthcare, Division of Nursing \\ Tokyo Healthcare University \\ Tokyo, Japan \\ y-takayama@thcu.ac.jp
}

\author{
Eiko Suzuki \\ Department of Nursing \\ International University of Health and Welfare \\ Tokyo, Japan \\ eikosuzuki@iuhw.ac.jp
}

Mayumi Sato

Department of Nursing, Faculty of Health \& Medical Care Saitama Medical University

Saitama, Japan

ma_sato@saitama-med.ac.jp

\begin{abstract}
The objective of this study was to determine a causal process underlying work engagement, in which individual resources (i.e., resilience) and job resources influence work performance, mediated by work engagement in different types of nurses working in long-term care contexts. We investigated a work engagement causal model in which individual and job resources were set as antecedent factors, work engagement as a mediating factor, and work performance as the outcome, to clarify differences between registered nurses (RNs) and licensed practical nurses (LPNs) working in long-term care contexts. We conducted a questionnaire-based survey with 1,786 Japanese nurses working in long-term care contexts in the Tohoku region. Using 1,269 respondents, we examined the causal model using structural equation modeling (SEM) and multiple population analysis to compare between RNs and LPNs. The results revealed a process whereby individual and job resources influenced work performance, mediated by work engagement, in RNs. In other words, greater individual and job resources enhance pride in work and positive emotion (i.e., work engagement), and greater positive emotion improves work performance. This process was not equivalent in LPNs. In LPNs, the most significant factor affecting work performance was the direct effect of job resources; moreover, the mediating effect of work engagement was not supported. The results demonstrated that in order to improve performance among LPNs working in long-term care contexts, it is important to provide job resource support, as well as to facilitate positive emotion through pride in one's work.
\end{abstract}

Keywords--work engagement; work performance; long-term care facility; registered nurses; licensed practical nurses

\section{INTRODUCTION}

In Japan, there are two types of professional nurses, with different educational backgrounds: registered nurses (RNs) and licensed practical nurses (LPNs). The Japanese LPN system was established in 1951 to respond to the shortage of nurses after World War II. At that time, although demand for nurses was increasing, the female high school entry rate was low, and it was difficult to train RNs sufficiently. Therefore, Japan created a system to ensure quality among nursing staff while reducing barriers to entry, creating the LPN status, which is conditional on graduating from junior high school [1, 2].

However, due to a rapidly aging population and recent medical progress, nurses today are required to have advanced abilities in autonomous assessment and performance. It may be a challenge for LPNs to acquire critical practical nursing skills, such as systematized understanding and assessment abilities, that correspond to the public's needs because the curriculum is limited to that which can be comprehended at the junior high school level.

The Japan Nursing Association aims to abolish the LPN status and unify it with RN training [2]. Moreover, in 1996, the Ministry of Health stated that, "We will try to integrate nurse training systems by the early stage of the 21 st century [3]."

In 2004, a system was implemented to allow LPNs to obtain RN qualifications, while working, via correspondence education. Thanks to such efforts, in recent years, the number of RNs has increased while the number of LPNs has decreased; more LPNs are acquiring RN qualifications and there are fewer LPN schools. Currently, there are 1,210,665 RNs and 347,675 LPNs in Japan [4]. The proportion of LPNs among all employed nursing staff is $28.7 \%$. As few LPNs are recruited to work in large hospitals, a significant number of today's LPNs are employed by small and medium-sized hospitals, clinics, and welfare facilities, such as nursing homes. 
In Japan, medical treatment for elderly patients who need long-term care is mainly carried out via long-term care beds. The number of nursing staff devoted to long-term care beds is about half that devoted to general beds, and the proportion of LPNs among long-term care nursing staff is larger than that among general beds, resulting in greater burden and stress for nursing staff in general $[5,6]$.

Building on recent developments in positive psychology, which focuses on using an individual's strengths to promote positive functioning, positive thinking has been introduced as a helpful means of managing workers' mental health [7, 8]. Work engagement, which was proposed by Schaufeli et al. [9], has gained attention as a principal research area focusing on the positive aspects of work.

Work engagement is a positive, affective-motivational state of fulfillment that is characterized by vigor, dedication, and absorption. Vigor refers to high levels of energy and resilience, willingness to invest effort in one's job, not being easily fatigued, and persistence in the face of difficulties. Dedication refers to a strong involvement in one's work, accompanied by feelings of enthusiasm and significance, and by a sense of pride and inspiration. Absorption refers to a pleasant state of total immersion in one's work that is characterized by time passing quickly and being unable to detach oneself from the job.

To date, work engagement has been proposed as a concept that indicates the well-being of workers, in contrast to burnout, which is an important research subject investigating burdens on workers' mental health. Previous studies have reported individual resources (e.g., emotional intelligence [10], individual job autonomy [11], positive affectivity [12]) and work resources (e.g., authentic leadership [13], organizational support, supervisor support, and social support [10]) as antecedent factors of work engagement. However, there is little outcome-focused research on the influence of work engagement on individuals and organizations, especially among LPNs.

This study aimed to examine a work engagement causal model in which individual resources (e.g., resilience) and job resources were set as antecedent factors, work engagement as a mediating factor, and work performance as the outcome, to clarify whether there were differences between RNs and LPNs working in long-term care contexts.

\section{METHODS}

\section{A. Study Design}

This quantitative cross-sectional study utilized an anonymous self-administered questionnaire.

\section{B. Participants}

We asked 140 hospitals with long-term care beds in 6 prefectures in the Tohoku region to participate in our study, and 79 of them agreed. The participants were 1,786 nurses working in long-term care contexts in the 79 hospitals.

\section{Survey Period}

September to November 2016

\section{Question Items and Measurement Instruments}

\section{1) Demographic Characteristics}

The survey included questions regarding the participants' gender, age, years of nursing experience, years of nursing in a long-term care context, type of nursing qualifications, employment type, and positions.

\section{2) Work Engagement}

We evaluated work engagement using the short form of the Japanese version of the Utrecht Work Engagement Scale (UWES-J), which was first developed by Schaufeli et al. [8] and translated into Japanese by Shimazu et al. [13]. The short form of the UWES-J has 9 items in 3 subscales: 3 vigor items, 3 dedication items, and 3 absorption items. All items are scored on a 7-point Likert-type scale ranging from 0 (never) to 6 (always). The range for each subscale score is from 0 to 18 , and the total score ranges from 0 to 54 , where higher scores indicate greater work engagement.

\section{3) Resilience}

Resilience was evaluated with the Bidimensional Resilience Scale developed by Hirano [15]. This scale consists of 21 items. Hirano regards resilience as comprising two separate factors: innate resilience, which is strongly related to congenital temperament, and acquired resilience, which can be acquired during development. The innate resilience subscale comprises 4 aspects: optimism, control, sociability, and vitality, while the acquired resilience subscale measures 3 aspects: problem solving, self-understanding, and understanding others. Items are rated on a 5-point Likert-type scale ranging from 1 (disagree) to 5 (agree). The innate resilience subscale consists of 12 items and the acquired resilience subscale consists of 9 items, with scores for each ranging from 1 to 5 points. The total scale score ranges from 1 to 5 points; higher scores indicate greater resilience.

\section{4) Job Resources}

Job resources were evaluated via level-based groupings: 8 items for task-level, 13 items for workgroup-level, and 7 items for organization-level, as per the short version of the New Brief Job Stress Questionnaire [16]. This instrument uses a 4-point response option: 1=Definitely, 2=Somewhat so, 3=Not exactly, and 4=Not at all. For the scale score, instead of summing the item scores, an average item score is calculated, ranging from 1 to 4 . Each scale (or item) score is converted so that higher scores indicate better states.

\section{5) Work Performance}

Work performance was evaluated through 3 items assessing performance of a duty, 3 items regarding realization of creativity, and 3 items related to active 
learning, as per the short version of the New Brief Job Stress Questionnaire [16]. This instrument also uses a 4point response option, corresponding to: 1=Definitely, $2=$ Somewhat so, $3=$ Not exactly, and $4=$ Not at all. For the scale score, instead of summing the item scores, an average item score is calculated, ranging from 1 to 4 . Each scale (or item) score is converted so that higher scores indicate better states.

\section{E. Analysis}

The analysis consisted of an evaluation of the hypothesized model (Fig. 1) using structural equation modeling (SEM), and an examination of configural and measurement invariances using multiple population analysis. IBM SPSS Statistics 24.0 and Amos Statistics 24.0 were used for the statistical analyses. The model fit was evaluated using the goodness-of-fit index (GFI), adjusted goodness-of-fit index (AGFI), root mean square error of approximation (RMSEA), and comparative fit index (CFI). A GFI of 0.9 or greater indicates a good model fit. RMSEA values of approximately 0.05 or less indicate a fair fit. Values less than 0.08 are still indicative of an acceptable fit, whereas values greater than 0.1 should lead to model rejection [17].

\section{F. Ethical Considerations}

Nursing directors of potential participating hospitals were contacted to request their cooperation; the study's purpose was explained both orally and in writing. The study objectives and methods were explained to the participating nurses in writing. This research was approved by the Ethics Committee of the International University of Health and Welfare, (No. 15-Ig-123, Jun 6, 2016）

\section{RESULTS}

\section{A. Descriptive Statistics}

We received responses from 1,432 nurses at 73 hospitals (collection rate: $80.2 \%$ ). Valid responses received from 1,269 nurses (valid response rate: $71.1 \%$ ) were analyzed. The participants comprised 79 male $(6.2 \%)$ and 1,190 female nurses $(93.8 \%)$; 843 were RNs $(66.4 \%)$, and 426 were LPNs (33.6\%). Demographics of the participants are shown in Table 1. Only $1.4 \%$ of LPNs held management positions, which was significantly less than the percentage of RNs in managerial roles.

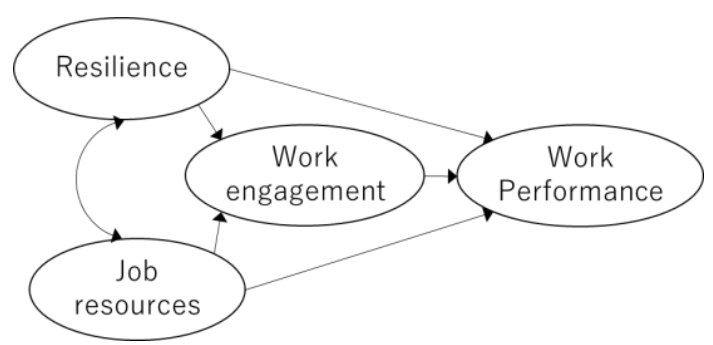

Figure 1. Hypothesized model
Participants' mean age was $44.0 \pm 11.7$ years; mean years of nursing experience were $20.9 \pm 11.8$ years and mean years of nursing in a long-term care context were $6.3 \pm 6.1$ years.

\section{B. Preliminary Analysis}

Independent t-tests were used to examine differences between RNs and LPNs in mean age, mean total scores, and mean subscale scores for resilience, work engagement, and work performance. Significant differences were found in age, work engagement total scores and subscale scores (i.e., absorption), job resources subscale scores (at the workgroup level), and work performance subscale scores (i.e., performance of a duty, realization of creativity, and active learning), as shown in Table 2.

In terms of work engagement, LPNs scores were significantly higher than RNs. In particular, LPNs scores were notably higher with regards to "absorption" as compared to RNs. In terms of work performance, LPNs scores were significantly higher than RNs for "performance of a duty" and "active learning"; however, RNs scores were notably higher for "realization of creativity."

\section{Relationships between Resilience, Job Resources, Work Engagement, and Work Performance}

Using SEM, we examined the hypothesized model, whereby resilience and job resources are mediated by work engagement to influence work performance. The fit of the model, shown in Fig. 2, was satisfactory, as demonstrated by the indices: $\mathrm{GFI}=0.951$, AGFI $=0.909$, $\mathrm{RMSEA}=0.079$, and $\mathrm{CFI}=0.936$. All of the path coefficients were significant $(p<$ $0.001)$.

\section{Multiple Population Analysis between RNs and LPNs}

Subsequently, we performed a multiple population analysis between RNs and LPNs to examine configural and measurement invariances. The model fit for both RN and LPN populations was favorable, as shown by the indices: GFI = 0.929, AGFI $=0.893$, RMSEA $=0.053$, and $\mathrm{CFI}=0.941$, demonstrating configural invariance. Therefore, we concluded that the same model applies to both RNs and LPNs.

\section{E. Measurement and configural invariance}

In addition, we examined the measurement invariance. After comparing the relevance of the six models, we concluded that the path coefficients from latent to observable variables are similar and path coefficients among latent variables are different; a weak measurement invariance was verified. In the figures, the latent and measurement variables are indicated as ellipses and rectangles, respectively.

Based on these results, we examined the path coefficients among latent variables. The model depicting RNs, indicated in Fig. 3, has a coefficient of determination, $R^{2}$, of $63 \%$ in work performance. The model for LPNs, in Fig. 4, has a coefficient of determination, $R^{2}$, of $52 \%$ in work performance. 
TABLE 1. DEMOgRAPHIC FACTORS ACCORDING TO TyPE OF NURSE

\begin{tabular}{|c|c|c|c|c|c|}
\hline \multirow[b]{2}{*}{ Variable } & \multicolumn{2}{|c|}{$\begin{array}{l}\text { Registered nurses (RNs) } \\
(n=843)\end{array}$} & \multicolumn{2}{|c|}{$\begin{array}{l}\text { Licensed practical } \\
\text { nurses (LPNs) } \\
(n=426)\end{array}$} & \multirow[t]{2}{*}{$\chi^{2}$-test } \\
\hline & $n$ & $\%$ & $n$ & $\%$ & \\
\hline \multicolumn{6}{|l|}{ Gender } \\
\hline Male & 56 & 6.6 & 23 & 5.4 & \\
\hline Female & 787 & 93.4 & 403 & 94.6 & \\
\hline \multicolumn{6}{|l|}{ Employment type } \\
\hline Full-time & 769 & 91.2 & 383 & 89.9 & \\
\hline Part-time & 74 & 8.8 & 43 & 10.1 & \\
\hline \multicolumn{6}{|l|}{ Position } \\
\hline Manager & 205 & 24.3 & 6 & 1.4 & $* * *$ \\
\hline Staff & 638 & 75.7 & 420 & 98.6 & \\
\hline
\end{tabular}

TABLE 2. DIFFERENCES AMONG VARIABLES BY TYPE OF NURSE

\begin{tabular}{|c|c|c|c|c|c|c|}
\hline \multirow[b]{2}{*}{ Variable } & \multirow[b]{2}{*}{ Range } & \multicolumn{2}{|c|}{$\begin{array}{l}\text { Registered nurses } \\
\text { (RNs) } \\
(n=843)\end{array}$} & \multicolumn{2}{|c|}{$\begin{array}{l}\text { Licensed practical } \\
\text { nurses (LPNs) } \\
\quad(n=426)\end{array}$} & \multirow[t]{2}{*}{$t$-test } \\
\hline & & Mean & $S D$ & Mean & $S D$ & \\
\hline Age & $20-70$ & 35.70 & 11.10 & 44.54 & 11.57 & $* * *$ \\
\hline Work Engagement & $0-54$ & 23.82 & 11.78 & 25.26 & 12.41 & $*$ \\
\hline Vigor & $0-18$ & 7.15 & 4.39 & 7.66 & 4.64 & \\
\hline Dedication & $0-18$ & 9.71 & 4.14 & 10.09 & 4.31 & \\
\hline Absorption & $0-18$ & 6.96 & 4.29 & 7.51 & 4.41 & $*$ \\
\hline Resilience & $1-5$ & 3.33 & 0.50 & 3.32 & 0.52 & \\
\hline Innate resilience factors & $1-5$ & 3.29 & 0.53 & 3.30 & 0.56 & \\
\hline Acquired resilience factors & $1-5$ & 3.37 & 0.54 & 3.34 & 0.55 & \\
\hline Job Resources & $1-4$ & 2.50 & 0.44 & 2.46 & 0.51 & \\
\hline Task-level & $1-4$ & 2.54 & 0.47 & 2.51 & 0.54 & \\
\hline Workgroup-level & $1-4$ & 2.62 & 0.54 & 2.55 & 0.60 & $*$ \\
\hline Organization-level & $1-4$ & 2.29 & 0.53 & 2.24 & 0.61 & \\
\hline Work Performance & $1-4$ & 2.50 & 0.46 & 2.52 & 0.48 & \\
\hline Performance of a duty & $1-4$ & 2.66 & 0.51 & 2.74 & 0.54 & $* *$ \\
\hline Realization of creativity & $1-4$ & 2.50 & 0.63 & 2.40 & 0.64 & $* *$ \\
\hline Active learning & $1-4$ & 2.35 & 0.61 & 2.42 & 0.62 & $*$ \\
\hline
\end{tabular}

$* p<0.05 ; * * p<0.01 ; * * * p<0.001 ; S D=$ standard deviation 


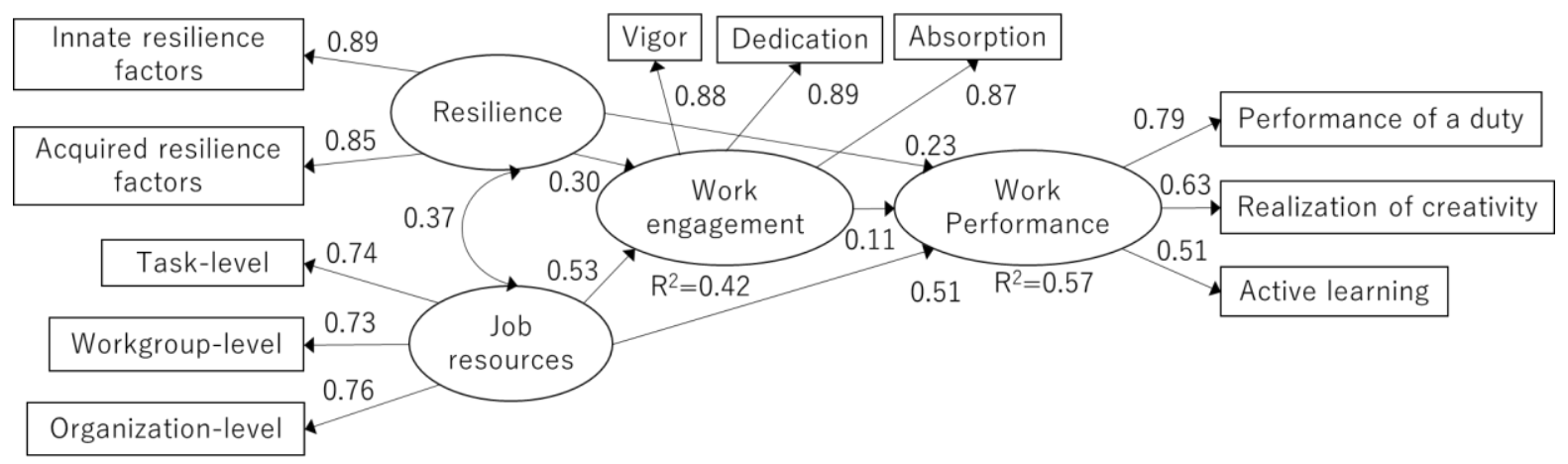

Figure 2. Causal model of resilience, job resources, work engagement, and work performance

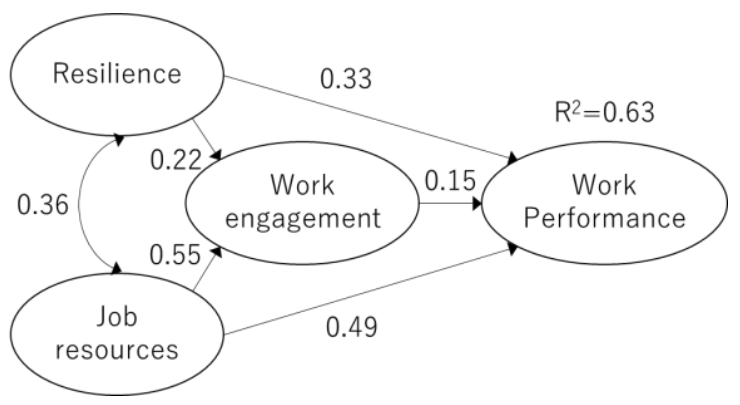

Figure 3. Causal model of registered nurses (RNs)

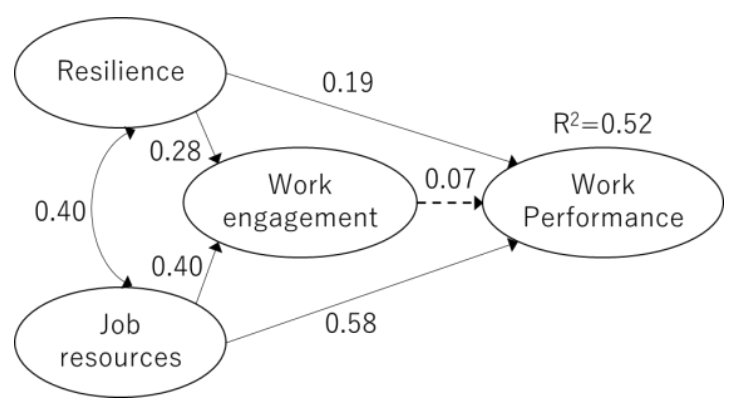

Figure 4. Causal model of licensed practical nurses (LPNs)

TABLE 3. Direct, InDiRect, AND Total EFFects of Work PERFormance by TyPe OF NURSE

\begin{tabular}{|c|c|c|c|c|c|c|}
\hline & \multicolumn{3}{|c|}{$\begin{array}{l}\text { Registered nurses (RNs) } \\
\qquad(n=843)\end{array}$} & \multicolumn{3}{|c|}{$\begin{array}{l}\text { Licensed practical nurses (LPNs) } \\
\qquad(n=426)\end{array}$} \\
\hline & $\begin{array}{c}\text { DIRECT } \\
\text { effects }\end{array}$ & $\begin{array}{l}\text { INDIRECT } \\
\text { effects }\end{array}$ & $\begin{array}{l}\text { TOTAL } \\
\text { effects }\end{array}$ & $\begin{array}{l}\text { DIRECT } \\
\text { effects }\end{array}$ & $\begin{array}{c}\text { INDIRECT } \\
\text { effects }\end{array}$ & $\begin{array}{l}\text { TOTAL } \\
\text { Effects }\end{array}$ \\
\hline Work Engagement & 0.15 & - & 0.15 & n.s & - & 0 \\
\hline Resilience & 0.33 & 0.03 & 0.36 & 0.19 & n.s & 0.19 \\
\hline Job Resources & 0.49 & 0.08 & 0.57 & 0.58 & n.s & 0.58 \\
\hline
\end{tabular}


As indicated in Fig. 3 and Table 3, among RNs, the path coefficient from resilience to work performance, which indicated a direct effect, was 0.33 , while the indirect effect of resilience to work performance, mediated by work engagement, was 0.03. Furthermore, the total effect (sum of direct and indirect effects), which demonstrated the mediating effect of work engagement, was 0.36 . In RNs, the path coefficient from job resources to work performance, which indicated a direct effect, was 0.49 , while the indirect effect of job resources to work performance, mediated by work engagement, was 0.08 . Furthermore, the total effect (sum of direct and indirect effects), which demonstrated the mediating effect of work engagement, was 0.57 .

On the other hand, as indicated in Fig. 4 and Table 3, among LPNs, the path coefficient from resilience to work performance, indicating a direct effect, was 0.19 . As the path coefficient from work engagement to work performance was not significant, the indirect effect of resilience on work performance, mediated by work engagement, was not proven. Consequently, the total effect (sum of direct and indirect effects), was equal to direct effect: 0.19. In LPNs, the path coefficient from job resources to work performance, which indicated a direct effect, was 0.58, while the indirect effect of job resources to work performance, mediated by work engagement, was not proven. Consequently, the total effect was equal to direct effect: 0.58 .

\section{DISCUSSION}

The results of this study indicated that in the process by which resilience and job resources influence work performance, the structure of the model is the same; however, there are observable differences between RNs and LPNs in terms of the causal process and strength of the causal relationship. The influence of work performance by resilience and job resources becomes greater as the process is mediated by work engagement in RNs. On the other hand, in LPNs, there was no mediating effect of work engagement. The most significant factor affecting work performance among LPNs was the direct effect of job resources.

As a result of the total effect, we observed that job resources had the greatest impact on work performance among both RNs and LPNs. However, differences between RNs and LPNs seem to exist in the form of processes affecting work performance and work engagement. In addition, when individual and job resources were set as antecedent factors, we observed other differences. In RNs, resilience notably affected work performance, but in LPNs, resilience only had a small effect on work performance. In other words, while among RNs, internal motivation factors such as resilience and work engagement mediate the engagement process, such that increasing these factors improves RNs' work performance, this is not the case for LPNs. This study suggests that LPNs' work performance is not mediated by internal motivation, but is directly affected by job resources.

RNs and LPNs working in long-term care contexts appear to have different processes that affect their engagement and performance; that is, the strength of the causal process of work engagement to work performance was different for each type of nurse. The key difference between RNs and LPNs, in this respect, is that LPNs do not demonstrate a significant change in work performance from work engagement. Thus, in LPNs, feeling proud of one's work and having positive emotions about work may not lead to enhanced work performance. However, LPNs were highly influenced by job resources, and it seems that the work environment and organizational context may directly affect their work performance. This study's results suggest that in order to improve the performance of LPNs working in long-term care, it is important to provide additional job resource support and not merely facilitate positive emotions through pride in one's work

The Japanese Nursing Association, an organization of professional nurses, has stated that "LPNs cannot advance in their careers," "LPNs complain about responsibility and being given the same task content as the RNs," and experience a "big disparity in salary compared to RNs [2]." According to a survey by Tanaka et al. [18], LPNs feel that there is no distinction between the duties of an RN and LPN; the LPNs are burdened by their lack of knowledge and the weight of responsibility. According to a job satisfaction survey [19], compared with RNs, LPNs have significantly lower scores for "salary" and "autonomy as professionals." In fact, LPNs receive lower salaries than RNs, and there is a difference of approximately 60,000 to 80,000 Japanese yen regardless of the age group [2]. From the results of these previous studies and the present study, it is suggested that work performance of LPNs is most strongly influenced by the workplace environment and organizational characteristics rather than positive feelings of internal motivation. Although LPNs are frequently responsible for similar tasks as RNs, they are regarded as lower in status and receive smaller salaries; it may be that LPNs feel frustrated by the fact that their efforts are not appreciated and rewarded. They may feel that the weight of their responsibility is heavy, but their knowledge is insufficient, creating a complex emotional and professional situation.

Clarifying the difference between the causal processes of work engagement among RNs and LPNs is a novel result of this study. Due to a rapidly aging society, advancing medical care practices, and increased complexity in the health care sphere, medical safety and team medicine are regarded as crucial in today's health care settings, and nursing staff must possess the ability to judge situations and act autonomously. However, the education of LPNs may be lacking because of the restrictions of the curriculum, which are set at the junior high school level. Thus, LPNs may experience difficulties in developing the skills needed to engage in logical assessment, autonomous care delivery, and systematic development of practical nursing skills [2]; this may impact their ability to provide quality care in response to emerging societal needs.

Long-term care contexts rely on a significant proportion of LPNs, yet little research, focused on the needs of this group of health care practitioners, has emerged. In this study, for the first time, we clarified the possibility that differences in educational backgrounds and qualifications may create a difference in the process of improving work performance. 
In the qualification of Japan's nursing staff, the double structure of RNs and LPNs is viewed as a problem. In order to further clarify influence factors, research on LPNs should be considered as an important social issue.

This study has several limitations. First, it was conducted in Tohoku region. It is necessary to expand the scope of the survey. Replication of the results in additional areas is necessary. Second, this was a cross-sectional study. As the next step, an examination of the related details through a longitudinal study, is recommended.

\section{CONCLUSIONS}

We examined the mediating effect of work engagement in resilience, job resources, and work performance in nurses working in long-term care contexts in Japan. When evaluating the hypothesized model, we established certain differences between RNs and LPNs. The model fit for both RN and LPN populations was favorable, demonstrating configural invariance. Therefore, we concluded that the structure of the model is the same, and the same model applies to both RNs and LPNs. However, we acknowledged the difference between the causal processes among the variables and the strength of the causal relationship. The results indicated that work performance is improved when work engagement, which is considered as a positive emotion toward work, is added to resilience, which is an individual resource, promoting work performance in RNs. On the other hand, in LPNs, there was no mediating effect of work engagement. Among this group of nurses, the most significant factor affecting work performance was the direct effect of job resources.

\section{ACKNOWLEDGMENTS}

We would like to express our sincere gratitude to the general nursing directors and nurses working in long-term care in the hospitals that participated in this study.

\section{REFERENCES}

[1] Y. Tsunoda, "The policy evolution of nurses in Japan: An economic analysis," Iryo To Shakai, vol. 6, no. 4, pp. 86-106, 1997 (in Japanese).

[2] Japanese Nursing Association. (2018). Efforts to Solve Problems of the Licensed Practical Nurse System [Online]. https://www.nurse.or.jp/nursing/kango_seido/index.html

[3] Ministry of Health, Labour and Welfare. (1996). Report on Study on Licensed Practical Nurses Problem [Online]. https://www.mhlw.go.jp/www1/topics/h12-kyoku_2/kenkou/tp01191e.html (in Japanese).

[4] Japanese Nursing Association, "Statistical Data on Nursing Service in Japan," 2015.

[5] S. Isokura, H. Kobayashi, T. Fukaya, A. Masuko, T. Hara, and H. Hashimoto, "Experiences of nurses who were transferred from general wards to long-term care wards," Jpn. J. Nurs. Manage., vol. 42, pp. 355-357, 2015 (in Japanese).

[6] K. Matoba, M. Sasaki, H. Ikeda, K. Ohata, and K. Nishizaka, "A survey on work-related stress in nurses and care workers for long-term care beds," Jpn. J. Gen. Nurs., vol. 38, pp. 484-486, 2007 (in Japanese).

[7] Y. Otsuka, "Theory of positive psychology and occupational mental health," Occup. Ment. Health, vol. 20(3), pp. 194-198, 2012 (in Japanese).
[8] A. Shimazu, "Work engagement: A new approach to positive psychology in the workplace," Job Stress Res., vol. 16(3), pp. 131-138, 2009 (in Japanese).

[9] W.B. Schaufeli, M. Salanova, V. González-Romá, and A.B. Bakker "The measurement of engagement and burnout: a two sample confirmatory factor analytic approach," J. Happiness Stud., vol. 3, pp. 71-92, 2002.

[10] Y. Freeney and M.R. Fellenz, "Work engagement as a key driver of quality of care: A study with midwives," J. Health Organ. Manag., vol. 27(3), pp. 330-349, 2013.

[11] M. Vera, I. M. Martínez, L. Lorente, and M. J. Chambel, "The role of co-worker and supervisor support in the relationship between job autonomy and work engagement among Portuguese nurses: A multilevel study," Soc. Indic. Res., vol. 126, pp. 1143-1156, 2016.

[12] J. H. M. Thian, P. Kannusamy, H. G. He, and P. Klainin-Yobas, "Relationships among stress, positive affectivity, and work engagement among registered nurses," Psychology, vol. 6(2), pp. 159-167, 2015.

[13] M. Bamford, C. Wong, and H. Laschinger, "The influence of authentic leadership and areas of work life on work engagement of registered nurses," J. Nurs. Manag., vol. 21(3), pp. 529-540, 2013.

[14] A. Shimazu, W. B. Schaufeli, S. Kosugi, A. Suzuki, H. Nashiwa, A Kato, et al. "Work engagement in Japan: Validation of the Japanese version of the Utrecht Work Engagement Scale," Appl. Psychol., vol. 57(3), pp. 510-523, 2008.

[15] M. Hirano, "A study of the classification of resilience factors: Development of the Bidimensional Resilience Scale (BRS),"Jpn. J. Pers., vol. 19(2), pp. 94-106, 2010 (in Japanese).

[16] A. Inoue, N. Kawakami, T. Shimomitsu, A. Tsutsumi, T. Haratani, T. Yoshikawa, et al., "Development of a short version of the New Brief Job Stress Questionnaire," Ind. Health, vol. 52(6), pp. 535-540, 2014.

[17] M. W. Browne and R. Cudeck, "Alternative ways of assessing model fit," in Testing Structural Equation Models, K. A. Bollen and J. S. Long, Eds. Newbury Park, CA: SAGE Publications, pp. 136-162, 1993.

[18] M. Tanaka and T. Miyaue, "Perceptions of careers, jobs, and nursing care: From a survey of nursing assistants working in social care and medical care settings," Bulletin of University of Kochi, 66, pp. 37-50, 2017 (in Japanese).

[19] H. Harima and S. Shono, "Relationship between job satisfaction and attributes of nurses working in long-term care beds," Papers Nurs. Assoc. Jpn.: Nurs. Manag., 42, pp. 416-419, 2012 (in Japanese).

\section{Chiaki Kinouchi, RN, PHN, PhD}

Chiaki Kinouchi is an associate professor in the Department of Nursing at Iwate University of Health and Medical Sciences, Iwate, Japan. She received her $\mathrm{PhD}$ in nursing from the International University of Health and Welfare Graduate School, Tokyo, Japan, in 2018.

\section{Eiko Suzuki, RN, PhD}

Eiko Suzuki is a professor at the International University of Health and Welfare Graduate School, Tokyo, Japan. She received her $\mathrm{PhD}$ in nursing from Tokyo Medical and Dental University, Tokyo, Japan, in 2010.

\section{Yuko Takayama, RN, PHN, PhD}

Yuko Takayama is an associate professor in the field of pediatric nursing at Tokyo Healthcare University, Tokyo, Japan. She received her $\mathrm{PhD}$ in nursing from the International University of Health and Welfare Graduate School, Tokyo Japan, in 2018. 
Mayumi Sato, RN, PHN, PhD

Mayumi Sato is a professor in the Department of Nursing, Faculty of Health \& Medical Care at the Saitama Medical University, Saitama, Japan. She received her PhD in nursing from the International University of Health and Welfare Graduate School, Tokyo Japan, in 2016. 